\title{
Characteristics of hospital admissions and invasive cardiology procedures in the Silesian Voivodeship in 2019 and 2020
}

\author{
Krystian Wita', Zbigniew Kalarus², Wojciech Wojakowski³ , Jacek Sikora ${ }^{4}$, Katarzyna Mizia-Stec', \\ Zbigniew Gąsior', Ewa Nowalany-Kozielska ${ }^{6}$, Krzysztof Gołba7 , Krzysztof Milewski ${ }^{8}$, Piotr Pączek, \\ Henryk Sobocik ${ }^{10}$, Jacek Olender ${ }^{11}$, Lucjan Szela"1 , Maciej Dyrbuś'12, Mariusz Gąsior ${ }^{13}$ \\ ${ }^{1} 1^{\text {st }}$ Department of Cardiology, School of Medicine in Katowice, Medical University of Silesia, Katowice, Poland \\ 2Department of Cardiology, Congenital Heart Diseases and Electrotherapy, Medical University of Silesia in Katowice, Zabrze, Poland \\ ${ }^{3}$ Department of Cardiology and Structural Heart Diseases, Medical University of Silesia, Katowice, Poland \\ ${ }^{4}$ Department of Cardiology, Silesian Centre for Heart Diseases, Medical University of Silesia, Zabrze, Poland \\ ${ }^{5}$ Department of Cardiology, School of Health Sciences, Medical University of Silesia, Katowice, Poland \\ ${ }^{6} 2^{\text {nd }}$ Department of Cardiology, School of Medicine with the Division of Dentistry in Zabrze, Medical University of Silesia, Zabrze, Poland \\ ${ }^{7}$ Department of Electrocardiology and Heart Failure, Medical University of Silesia, Katowice, Poland \\ ${ }^{8}$ Cardiology and Cardiac Surgery Center, Center for Cardiovascular Research and Development, American Heart of Poland, BielskoBiała, Poland \\ 'Department of Cardiology, Public Clinical Hospital, Sosnowiec, Poland \\ ${ }^{10}$ Voivodeship Specialist Hospital No. 2, Jastrzębie-Zdrój, Poland \\ "Scanmed, Kraków, Poland \\ ${ }^{12 S t u d e n t ~ S c i e n t i f i c ~ S o c i e t y, ~} 3^{\text {rd }}$ Department of Cardiology, School of Medical Sciences in Zabrze, Medical University of Silesia, Katowice, Poland \\ ${ }^{13} 3^{\text {rd }}$ Department of Cardiology, School of Medical Sciences in Zabrze, Medical University of Silesia, Katowice, Poland
}

\author{
Correspondence to: \\ Maciej Dyrbuś, MD, \\ Student Scientific Society, \\ $3^{\text {rd }}$ Department of \\ Cardiology, \\ School of Medical Sciences \\ in Zabrze, Medical \\ University of Silesia, \\ Silesian Center for Heart \\ Diseases, \\ Skłodowskiej Curie 9 \\ 41-800 Zabrze, Poland, \\ phone: +48 323733860 , \\ e-mail: mdyrbus@op.pl \\ Copyright by the \\ Author(s), 2021 \\ Kardiol Pol. 2021; \\ 79 (9): 1022-1024: \\ DOI: 10.33963/KP.a2021.0077 \\ Received: \\ April 8, 2021 \\ Revision accepted: \\ July 26, 2021 \\ Published online: \\ July 28, 2021
}

\section{INTRODUCTION}

The rapid spread of the coronavirus disease (COVID-19) has imposed significant changes for health care systems [1]. In order to provide care for patients infected with severe acute respiratory syndrome coronavirus 2 (SARS-CoV-2) and to reduce the risk of viral transmission, the work of many cardiology departments and outpatient clinics had to be reorganized, while many wards have been repurposed to facilitate care for patients infected with SARS-CoV-2 [2,3]. Many planned hospital admissions have been postponed or canceled, while some patients resigned from a planned admission [4].

Due to the fear of becoming infected, many patients were reluctant to present in the hospital or call for an ambulance, which often resulted in a delay in the contact with healthcare in acute cases.

The purpose of the present analysis was to summarize the causes of hospital admissions and the major procedures performed in cardiology departments in the Silesian Voivodeship in 2020, with reference to the respective data from 2019.

\section{METHODS}

The analysis was based on data acquired from the questionnaires provided annually by the cardiology wards in the Silesian Voivodeship to the provincial consultant in cardiology. The response to the questionnaires is obligatory for every unit in the Voivodeship. The questionnaires contain information regarding the number of hospital admissions with the main diagnoses, along with the data on the procedures performed during these admissions. In the present study, the data regarding the years 2019 and 2020 were analyzed. It should be noted that the study encompassed the entire years and the detailed data for specific months, as well as the short- or long-term outcomes of the treated patients, were not available for the purpose of this analysis.

The approval of the ethics committee, and patient informed consent were not required for the purpose of this study.

\section{Statistical analysis}

The results presented in the manuscript were expressed as numbers and percentages. The comparison of the analyzed variables for each year was presented as relative differences.

\section{RESULTS AND DISCUSSION}

In 2019, there were 61537 hospital admissions to the cardiology departments, which decreased by $22.4 \%$ to 47734 in 2020 . The 


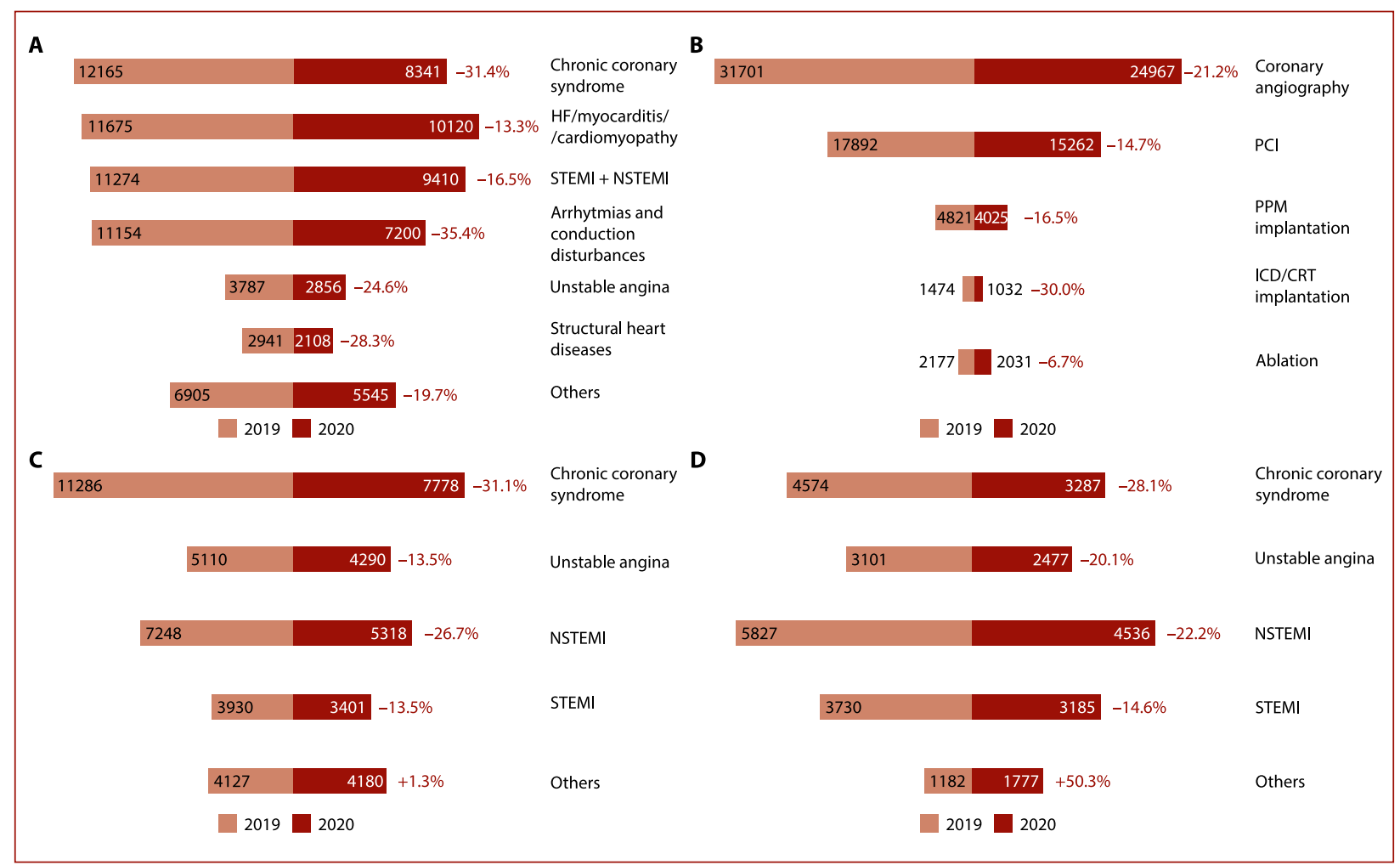

Figure 1. Data regarding the number of hospital admissions and invasive cardiology procedures in 2019 and 2020 in the Silesian Voivodeship. A. The primary causes of admissions. B. The number of invasive procedures. C. The number of coronary angiographies with regard to the indication. $\mathbf{D}$. The number of percutaneous coronary interventions with regard to the indication. The percentage changes between the respective values are described in red

Abbreviations: CRT, cardiac resynchronization therapy device; HF, heart failure; ICD, implantable cardioverter-defibrillator; NSTEMI, non-ST-segment-elevation myocardial infarction; PCl, percutaneous coronary intervention; PPM, permanent pacemaker; STEMI, ST-segment-elevation myocardial infarction

major diagnoses in the respective years are summarized in Figure $1 \mathrm{~A}$. The primary causes of admissions in 2019 were chronic coronary syndromes (CCS) $(19.8 \% ; n=12$ 165) and heart failure (HF), myocarditis, or cardiomyopathies (19.0\%; $n=11$ 675). In 2020 the most prevalent cause of admission was $\mathrm{HF}$, myocarditis or cardiomyopathies (21.2\%; $\mathrm{n}=10120)$ followed by myocardial infarction (MI) (19.7\%; $n=9410)$.

There was a reduction in the number of all analyzed invasive procedures between 2020 and 2019, as presented in Figure 1B. The number of coronary angiography procedures decreased by $21.4 \%$, while the number of percutaneous coronary interventions $(\mathrm{PCI})$ decreased by $14.6 \%$. The numbers of cardiac ablations or implantations of a permanent pacemaker, implantable cardioverter defibrillator, or cardiac resynchronization therapy devices were also reduced when compared with 2019.

As demonstrated in Figure 1C, in 2019 patients underwent coronary angiography most frequently due to CCS (35.6\%; $n=11286)$ and non-ST-segment elevation myocardial infarction (NSTEMI) $(22.9 \% ; n=7248)$, which remained the most prevalent indications in 2020 (31.2\%; $\mathrm{n}=7778$ and 20.6\%; $\mathrm{n}=5138$, respectively). In 2019 the majority of PCls were performed due to NSTEMI (32.6\%; $\mathrm{n}=5827)$ and CCS (25.6\%; $\mathrm{n}=4574)$, as described in Figure 1D. In 2020 the most prevalent indications for $\mathrm{PCl}$ were NSTEMI (29.7\%; $n=4536)$, followed by ST-segment elevation myocardial infarction (STEMI) $(20.9 \% ; n=3185)$.

In the Silesian Voivodeship, the number of patients admitted to the cardiology departments has seen a major reduction, which has also exerted a drop in the number of invasive procedures performed from the outbreak of the pandemic. A similar trend has been observed in Europe and the United States, where in the initial phase of the SARS-CoV-2 spread, the number of acute cardiovascular hospitalizations was reduced by almost $45 \%[1,5-7]$.

The decrease in the number of admissions in the analyzed period has been observed regardless of the cause and diagnosis but was numerically most prominent in patients with CCS (by 31.4\%, a reduction of 3824 admissions). The necessity to reorganize the cardiology departments, the redistribution of ward employees and the division of the departments to separate the patients with regard to their infectious status could play a role in this decrease.

Moreover, similarly to the specialties, the reduction in the number of planned admissions has been recommended in order to secure hospital beds for patients with life-threatening conditions. 
A major reduction has also been observed in the numbers of patients with acute coronary syndromes, which corresponds with similar observations from Europe and the United States [5-9]. It is worth mentioning that the percentage of patients with $\mathrm{Ml}$ undergoing invasive diagnostics and treatment remained similar, as $82.0 \%$ of patients with $\mathrm{MI}$ in 2020 and $84.8 \%$ in 2019 underwent $\mathrm{PCl}$.

Finally, despite an absolute reduction in the numbers of admissions when compared with 2019, HF, myocarditis, or cardiomyopathy became the primary cause of hospital admissions in 2020 , constituting more than $20 \%$ of all hospitalizations. One of the hypotheses explaining the increase in the relative frequency of admissions due to HF could be limited access to ambulatory care, provided mostly as the telehealth visits, or the apprehension of patients of becoming infected when seeking medical advice. Moreover, although the stay-at-home campaigns aimed to substantially reduce the social risk of viral transmission, the tendency to isolate at home could potentially lead to patients deferring seeking medical contact.

An effective HF treatment is based on dynamic responses to the patients' conditions, for instance using day-care units, which significantly reduced the risk of recurrent hospitalizations in patients with HF before the pandemic [10]. After the outbreak of COVID-19, the extensiveness of the functioning of these facilities has been reduced or even suspended.

Taking into consideration the absolute decrease in the number of hospital admissions due to HF and the previously published data on the increase of life-threatening arrhythmias in remotely monitored patients with $\mathrm{HF}$, one can speculate that the fear of seeking medical care could have resulted in more pronounced development of $\mathrm{HF}$, potentially leading to the death of some patients before arrival to the hospital $[11,12]$.

It is worth noting that an approximate $16 \%$ reduction in the number of admissions due to HF or acute coronary syndrome is lower than in the prior analyses [5-9]. However, the results of this analysis encompass the entire years, including the first months of 2020 before the pandemic, when the healthcare system has functioned similarly as in 2019.

In conclusion, in 2020 in the Silesian Voivodeship, there has been a decline in the number of hospital admissions to cardiology departments regardless of the condition, which has been followed by a reduction in the number of invasive procedures performed when compared with 2019. Unfortunately, the number of patients referred to the cardiology departments with acute coronary syndromes decreased in comparison with 2019, however, their treatment remained similar as before the pandemic.

\section{Article information}

Conflict of interest: None declared.

Open access: This article is available in open access under Creative Common Attribution-Non-Commercial-No Derivatives 4.0 International (CC BY-NC-ND 4.0) license, allowing to download articles and share them with others as long as they credit the authors and the publisher, but without permission to change them in any way or use them commercially. For commercial use, please contact the journal office at kardiologiapolska@ptkardio.pl.

How to cite: Wita K, Kalarus Z, Wojakowski W, et al. Characteristics of hospital admissions and invasive cardiology procedures in the Silesian Voivodeship in 2019 and 2020. Kardiol Pol. 2021; 79(9): 1022-1024, doi: 10.33963/KP.a2021.0077.

\section{REFERENCES}

1. Sokolski M, Gajewski P, Zymliński R, et al. Impact of coronavirus disease 2019 (COVID-19) outbreak on acute admissions at the emergency and cardiology departments across Europe. Am J Med. 2021; 134(4): 482-489, doi: 10.1016/j.amjmed.2020.08.043.

2. Her M. Repurposing and reshaping of hospitals during the COVID-19 outbreak in South Korea. One Health. 2020; 10: 100137, doi: 10.1016/j.onehlt.2020.100137, indexed in Pubmed: 32373705.

3. Świerad M, Dyrbuś K, Szkodziński J, et al. Telehealth visits in a tertiary cardiovascular center as a response of the healthcare system to the severe acute respiratory syndrome coronavirus 2 pandemic in Poland. Pol Arch Intern Med. 2020; 130: 700-703.

4. The number of admissions in respective months of 2020 with reference to 2019 obtained from the National Health Fund. https://zdrowedane. nfz.gov.pl/course/view.php?id=139 (March 8, 2021).

5. De Filippo O, D'Ascenzo F, Angelini F, et al. Reduced rate of hospital admissions for ACS during Covid-19 outbreak in Northern Italy. N Engl J Med. 2020; 383(1): 88-89, doi: 10.1056/NEJMc2009166, indexed in Pubmed: 32343497.

6. Bhatt AS, Moscone A, McElrath EE, et al. Fewer hospitalizations for acute cardiovascular conditions during the COVID-19 pandemic. J Am Coll Cardiol. 2020; 76(3): 280-288, doi: 10.1016/j.jacc.2020.05.038, indexed in Pubmed: 32470516.

7. Gąsior M, Gierlotka M, Tycińska A, et al. Effects of the coronavirus disease 2019 pandemic on the number of hospitalizations for myocardial infarction: regional differences. Population analysis of 7 million people. Kardiol Pol. 2020; 78(10): 1039-1042, doi: 10.33963/KP.15559, indexed in Pubmed: 32820878.

8. Hawranek M, Grygier M, Bujak K, et al. Characteristics of patients from the Polish Registry of Acute Coronary Syndromes during the COVID-19 pandemic: the first report. Kardiol Pol. 2021; 79(2): 192-195, doi: 10.33963/KP.15756, indexed in Pubmed: 33463992.

9. Metzler B, Siostrzonek P, Binder RK, et al. Decline of acute coronary syndrome admissions in Austria since the outbreak of COVID-19: the pandemic response causes cardiac collateral damage. Eur Heart J. 2020; 41(19): 1852-1853, doi: 10.1093/eurheartj/ehaa314, indexed in Pubmed: 32297932.

10. Gąsior M, Niedziela JT, Sikora J, et al. Improved prognosis in patients with recurrent hospitalizations for heart failure after day-care management. Kardiol Pol. 2019; 77(10): 975-977, doi: 10.33963/KP.14987, indexed in Pubmed: 31553331.

11. Tajstra $M$, Kurek A, Pyka $\measuredangle$, et al. The increased rate of life-threatening interventions in remotely monitored patients with heart failure during the coronavirus disease 2019 pandemic. Pol Arch Intern Med. 2020; 130(10): 913-914, doi: 10.20452/pamw.15505, indexed in Pubmed: 32643914.

12. Marijon E, Karam N, Jost D, et al. Out-of-hospital cardiac arrest during the COVID-19 pandemic in Paris, France: a population-based, observational study. Lancet Public Health. 2020; 5(8): e437-e443, doi: 10.1016/S24682667(20)30117-1, indexed in Pubmed: 32473113. 